\title{
BMJ Open Does employee participation in workplace health promotion depend on the working environment? A cross-sectional study of Danish workers
}

\author{
Marie Birk Jørgensen, ${ }^{1}$ Ebbe Villadsen, ${ }^{1}$ Hermann Burr, ${ }^{2}$ Laura Punnett, ${ }^{3}$ \\ Andreas Holtermann ${ }^{1,4}$
}

To cite: Jørgensen MB, Villadsen $\mathrm{E}, \mathrm{Burr} \mathrm{H}$, et al. Does employee participation in workplace health promotion depend on the working environment? A cross-sectional study of Danish workers. BMJ Open 2016;6: 010516.

doi:10.1136/bmjopen-2015010516

- Prepublication history for this paper is available online. To view these files please visit the journal online (http://dx.doi.org/10.1136/ bmjopen-2015-010516).

Received 10 November 2015 Revised 30 March 2016 Accepted 25 April 2016
CrossMark

For numbered affiliations see end of article.

Correspondence to Dr Marie Birk Jørgensen; mbj@nrcwe.dk

\section{ABSTRACT}

Objectives: To investigate if participation in workplace health promotion (WHP) depends on the work environment.

Methods: Questionnaire data on participation in WHP activities (smoking cessation, healthy diet, exercise facilities, weekly exercise classes, contact with health professionals, health screenings) and the work environment (social support, fatiguing work, physical, quantitative and emotional demands, job control and WHP availability setting) were collected crosssectionally in 2010 in a representative sample $(n=10605)$ of Danish workers. Binary regression analyses of the association between work environment characteristics and participation in WHP were conducted and adjusted for age, gender and industry.

Results: WHP offered during leisure time was associated with lower participation in all measured activities compared with when offered during working hours. Low social support and fatiguing work were associated with low participation in WHP. No associations with participation in WHPs were observed for physical work or quantitative demands, work pace or job strain. However, high physical demands/low job control and high emotional demands/low job control were associated with low participation.

Conclusions: Lower participation in WHP was associated with programmes during leisure, low social support, very fatiguing work and high physical or emotional demands with low job control. This suggests that to obtain proper effect of health promotion in a workplace setting, a good work environment is essential.

\section{INTRODUCTION}

Non-communicable diseases such as diabetes, heart disease and cancer are prevalent and increasing in Western countries. ${ }^{1}$ These diseases have large consequences for the individual's quality of life and function and for societal costs to healthcare and productivity loss. ${ }^{1}$ The causes of these diseases are
Strengths and limitations of this study

- Involves a large number of employees from several different occupations and industries in a representative sample of Danish employees.

- Self-reported information regarding work environment and the availability of health promotion represents the perceptions of the participants, which is known to impose risks of bias.

- The reporting of participation reflects any level of participation in health promotion within the last year.

- All data were collected concurrently and analyses performed in a cross-sectional design, which hampers interpretation of causality.

mainly related to lifestyle such as poor diet, physical inactivity and smoking, ${ }^{1}$ and also due to working environment features (ie, high physical work demands) ${ }^{2}$ and stress due to psychosocial job features. ${ }^{3} 4$

The workplace has therefore been suggested to be a suitable setting for health promotion. The suggestion is based on the notion that health promotion requires not just behaviour change but also a supportive environment. Thus, safe, stimulating, satisfying and enjoyable working conditions are meant to support health-promoting activities. ${ }^{5}$ A recent meta-analysis concluded that workplace health promotion (WHP) is primarily effective among white-collar workers and highly selected individuals. ${ }^{6}$ Many WHP studies report low participation rates $^{7}$ and problems with implementation and sustainability associated with the organisation of the work. ${ }^{8-10}$ Thus, the workplace may only be a suitable setting for health promotion under good work environment conditions.

Most likely, WHP interventions have been initiated without ensuring proper contextual work environment. The socioecological 
framework has been used theoretically to illustrate how the work environment may limit participation in WHP. ${ }^{11}$ Factors that may impact individuals' participation involve structural (ie, quantitative demands, physical demands and organisation of the work) ${ }^{12}$ and interpersonal factors (ie, social support). ${ }^{13-15}$ For example, low job control may decrease the possibility to organise one's work to be able to participate in activities and WHP during paid working hours rather than during leisure may decrease barriers related to leisure time duties. However, it remains to be established how these factors are associated with participation in WHP.

Therefore, the aim of this study was to investigate the association between organisational, structural and interpersonal factors in the work environment and participation in WHP in a large representative sample of Danish employees. The following hypotheses were tested: (1) WHP offered within working hours is associated with higher participation than WHP offered outside working hours, (2) structural factors of the job and the work environment (high quantitative, physical and emotional work demands, low job control and fatiguing work) are associated with lower participation in WHP and (3) interpersonal factors in the working environment (ie, low social support) are associated with lower participation in WHP.

\section{STUDY POPULATION}

In 2010, the fifth round of the Danish Work Environment Cohort Study (DWECS) was conducted. ${ }^{16}$ This DWECS round featured a random sample of $\sim 21000$ workers aged 18-59 years drawn from the Central Population Register of Denmark; of these, 53\% (10 605) participated in the survey. Paper questionnaires were sent to their personal addresses and participants were asked to reply either to the paper questionnaire or to use a link for electronic response.

\section{METHODS}

Participants responded to a self-administered questionnaire with items regarding availability and participation in WHP activities as well as features of the work environment (physical work demands, physically fatiguing work, quantitative demands, emotional demands, social support from colleagues and supervisor and job control).

\section{Independent variables}

WHP availability

Availability of WHP programmes was determined by the question (modified from Grosch et $a l^{17}$ ) and previously reported in Jorgensen et al: ${ }^{16}$ 'During the last year, have you been offered health promotion via your workplace?' with the response categories 'No', 'Yes, during working hours' and 'Yes, outside working hours'. The following six types of WHP were covered: smoking cessation, healthy diet, exercise facilities, weekly exercise classes, contact with health professionals (physiotherapy, psychologist or the like) and health screenings. Availability timing (during working hours vs outside working hours (termed leisure time)) was asked for each specific activity.

\section{Physical work demands}

Physical work demands were measured by 10 items on the typical duration of physical postures and activities at work: 'Does your job require that...' 'you are sitting down?'; 'you are standing at the same place?'; 'you work with your back bent...forward without supporting with your arms or hands?'; 'you twist or bend your back many times per hour?'; 'you lift your arms at or above shoulder height?'; 'you do the same finger movements many times per minute (ie, typing work)?', 'you do the same arm movements many times per minute (ie, packing work, mounting, machine feeding, cutting)?'; 'you squat or kneel, when you work?', 'you push or pull?', 'you lift or carry?'. Answer categories were given points corresponding to: almost all the time (100), approximately three-fourth of the time (75), approximately half of the time (50), approximately one-fourth of the time (25), seldom/very little (6), never (0). Answer categories for the item for sitting time was reversed. The sum of all physical work demand scores was calculated and categorised into quartiles of the total score.

\section{Fatiguing work}

Fatiguing work was measured with a question inspired by the Need for Recovery scale: ${ }^{18} \quad 19$ 'How physically exhausted generally in your body are you after a typical work day?'. The six answer categories were categorised into three levels of fatigue: not fatigued (not exhausted, a little bit exhausted), moderately fatigued (somewhat exhausted) and very fatigued (very exhausted, totally exhausted).

\section{Quantitative demands}

Quantitative demands were determined by three questions replied to on one scale (always, often, sometimes, rarely, never/almost never): 'How often...' '...is your work unevenly distributed, so that it piles up?'; '...do you not have time to complete all your work tasks?' and '...do you have to do overtime?'. Each answer was converted to a scale from 0 to 100 at equal 20-point increments $(0=$ never, $100=$ always $)$. The mean of these was generated by dividing the sum of the items by the number of items, and this variable was dichotomised at the median value into high/low.

\section{Work pace}

Work pace was determined by the question: 'How often do you have to work very fast?' (always, often, sometimes, rarely, never/almost never). The answer was converted to a scale from 0 to 100 at equal 20-point increments $(0=$ never, $100=$ always $)$ and dichotomised at the median value into high/low. 


\section{Emotional demands}

Emotional demands was measured with three questions with one answer scale (to a very high degree, to a high degree, partly, to a low degree, to a very low degree): 'To what degree...' '.... is your work emotionally demanding?'; '...do you get emotionally involved in your work?' and '...do you have to deal with other people's problems at work?'. The answer was converted to a scale from 0 to 100 at equal 20-point increments $(0=$ to a very low degree, $100=$ to a very high degree) and dichotomised at the median value into high/low.

\section{Control}

Control was determined by two items regarding influence: 'Are you involved in the planning of your work (ie, how it's done or who you work with)?' (always, usually, usually not, never) and 'Do you have a large degree of influence concerning your work?' (always, often, sometimes, rarely, never/almost never). The answers were converted to a scale from 0 to 100 at equal 25-point increments $(0=$ never, $100=$ always $)$ for the question regarding involvement in planning and at equal 20-point increments for the question regarding influence $(0=$ never/almost never, $100=$ always ) and dichotomised at the median value into high/low.

\section{Job demands/control ratios}

Three ratios were computed to represent the effect of qualitatively different types of job demands, relative to the degree of decision latitude available to choose how to respond to those demands. Thus the numerator varied but the denominator in each case was the job control scale above. Psychosocial job strain was the ratio of quantitative demands to control, dichotomised into high/low at the median value. Physical demands and control ratio: The ratio of physical work demands (above score from 0 to 100) to job control was calculated. Emotional demands and control ratio: The ratio of emotional demands to control was calculated with emotional demands in the nominator and control in the denominator.

\section{Social support}

Social support was measured with four questions on one answer scale (always, often, sometimes, rarely, never/ almost never, not relevant): 'How often...' '....are your colleagues willing to listen to your problems at work?'; '...do you get help and support from your colleagues?'; '....is your nearest supervisor willing to listen to your problems at work?'; '...do you get help and support from your nearest supervisor?'. The answers were converted to a scale from 0 to 100 at equal 20-point increments $(0=$ never $/$ almost never, $100=$ always $)$ and dichotomised at the median value into high/low.

\section{Dependent variables}

Participation in WHP

Participation in WHP was calculated only among those who reported to have it available and was determined by the question 'Have you used it [the specific WHP activity asked for availability of]? (if you did, please mark)'. The following six WHP activities were requested: 'smoking cessation', 'healthy diet', 'exercise facilities', 'weekly exercise classes', 'contact with health professionals (physiotherapist, psychologist or the like)' and 'health screenings'.

\section{Covariates}

Age and gender were obtained from the Central Population Register and industry from Statistics Denmark's registers. Age was categorised into the following categories: 18-29, 30-39, 40-49 and 50-59 years. Industry was categorised into: manufacturing, construction, graphics, transportation and retail, trading, service, agriculture, social and healthcare, teaching and research, finance/public administration and business administration.

\section{Statistical analyses}

Spearman's correlation coefficients were calculated for all pairs of independent variables. Variables with high collinearity were either collapsed or presented in separate models. Binomial logistic regression models were used to estimate ORs for participation in WHP according to the work environment factors. Job strain, emotional demands/control ratio, social support, fatigue, physical work demands and availability timing were added into the same regression model to investigate their mutually adjusted associations with participation. The ratio of physical work demands/job control was investigated in a separate model with the same covariates, where it replaced the other two ratios.

All models were adjusted for age, gender and industry. Furthermore, the model investigating participation in smoking cessation was restricted to those with current/ previous smoking status. Statistical analysis was performed using the SAS statistical software 9.2 for Windows. An $\alpha$ level of 0.05 was defined as representing statistical significance.

\section{RESULTS}

The two social support indices (ie, colleague support and supervisor support) were highly correlated (table 1) and therefore collapsed. Physical work demands and lifting and carrying were highly correlated, and therefore we moved on with the measure of physical work demands only. There were 9835 (93\%) employees providing data on availability and participation of WHP included in the analyses.

A little more than half of the study population was men $(54 \%)$ and the most prevalent age groups were adults aged $40-49$ years $(32 \%)$. Further descriptive information on 


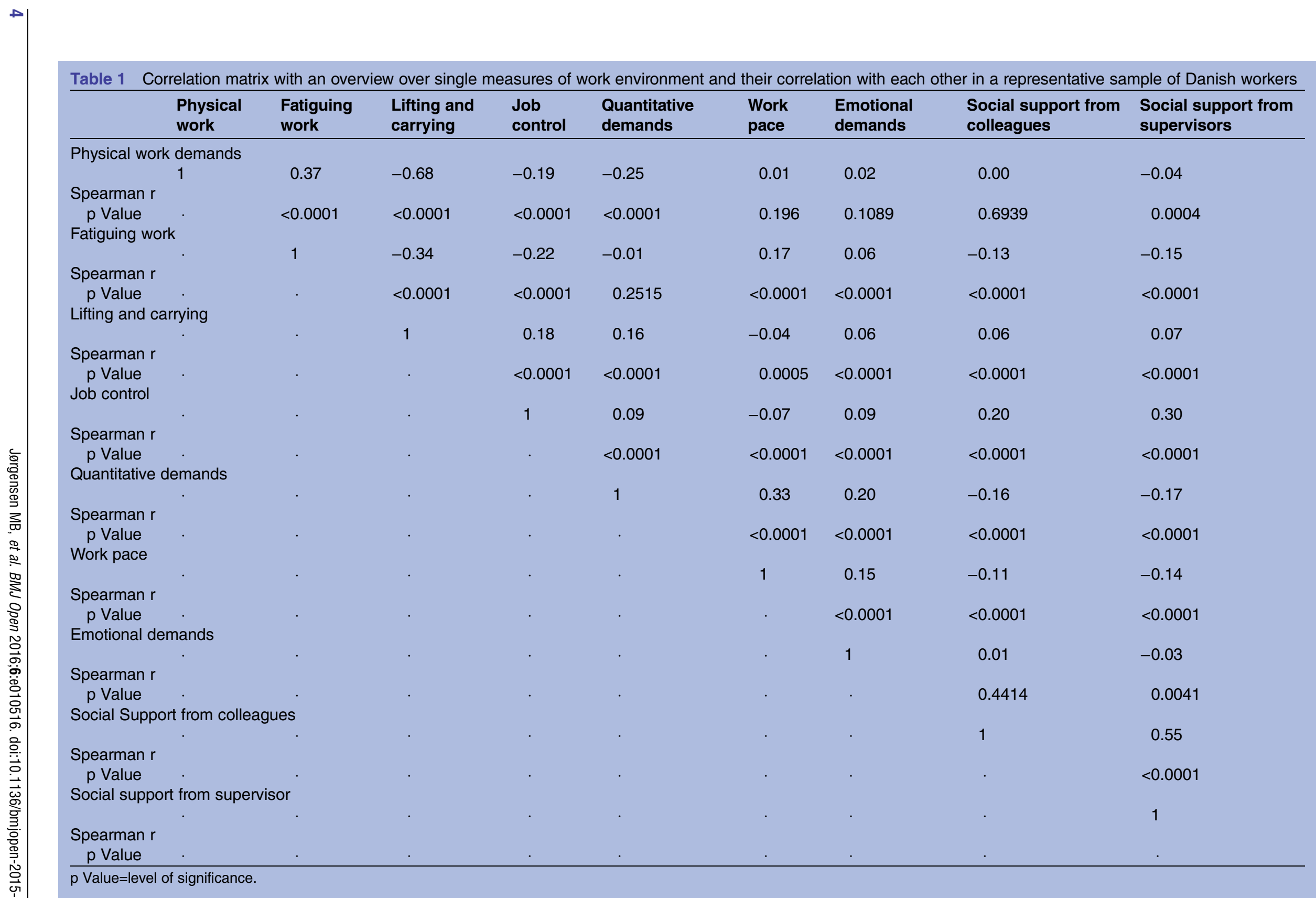


gender distribution, age group, industry (30\% unknown), work environment and availability of health promotion is presented in table 2. The most prevalent available WHP was contact to health professional $(33 \%)$ and the least prevalent WHP available in the population was smoking cessation (16\%). Among those with WHP available, the WHP with the highest participation was healthy diet (53\%) and the WHP with the lowest participation was smoking cessation (10\%). Further descriptive detail regarding availability and participation is given in table 3 .

\section{The association between work environment and participation}

The results of the regression models are presented in table 4 and are described below.

\section{Availability timing}

Availability timing of WHP during working hours versus leisure time was highly associated with participation in

Table 2 Descriptive information regarding distribution of age, gender, industry and work environment in a representative sample of Danish workers

\begin{tabular}{|c|c|c|}
\hline $\mathrm{N}=9835$ & $\mathbf{n}$ & Per cent \\
\hline \multicolumn{3}{|l|}{ Gender (\%) } \\
\hline Women & 3925 & 45.9 \\
\hline Men & 4627 & 54.1 \\
\hline \multicolumn{3}{|l|}{ Age (\%) } \\
\hline $18-29$ years & 1429 & 16.7 \\
\hline 30-39 years & 1944 & 22.7 \\
\hline $40-49$ years & 2703 & 31.6 \\
\hline 50-59 years & 2476 & 29.0 \\
\hline \multicolumn{3}{|l|}{ Industry (\%) } \\
\hline Manufacturing (\%) & 910 & 10.6 \\
\hline Construction (\%) & 244 & 2.9 \\
\hline Graphics (\%) & 64 & 0.7 \\
\hline Transportation and retail (\%) & 621 & 7.3 \\
\hline Trading (\%) & 243 & 2.8 \\
\hline Service (\%) & 392 & 4.6 \\
\hline Agriculture (\%) & 57 & 0.7 \\
\hline Social and healthcare (\%) & 1683 & 19.7 \\
\hline Teaching and research (\%) & 684 & 8.0 \\
\hline Finance/public administration (\%) & 629 & 7.4 \\
\hline Business administration (\%) & 425 & 5.0 \\
\hline Unknown & 2600 & 30.4 \\
\hline High emotional demands & 4940 & 51.6 \\
\hline High emotional demands/control ratio & 4641 & 49.9 \\
\hline Low social support & 4306 & 45.8 \\
\hline High physical work demands & 4769 & 50.0 \\
\hline High job strain & 4639 & 49.7 \\
\hline High quantitative demands & 5146 & 53.6 \\
\hline High work pace & 6477 & 67.7 \\
\hline High physical work demands/control & 4581 & 49.9 \\
\hline Low control & 5723 & 60.1 \\
\hline \multicolumn{3}{|l|}{ Fatigue (\%) } \\
\hline No & 5971 & 62.3 \\
\hline Moderate & 2594 & 27.1 \\
\hline Very & 1023 & 10.7 \\
\hline
\end{tabular}

Table 3 Availability and participation during the past year for six different categories of health promotion among a representative sample of Danish workers

\begin{tabular}{|c|c|c|c|c|}
\hline & \multicolumn{2}{|c|}{ Availability } & \multicolumn{2}{|c|}{ Participation } \\
\hline & $\mathbf{n}$ & $\begin{array}{l}\text { Per } \\
\text { cent }\end{array}$ & $\mathbf{n}$ & $\begin{array}{l}\text { Per } \\
\text { cent }\end{array}$ \\
\hline Smoking cessation & 1600 & 16.3 & 156 & 9.8 \\
\hline Healthy diet & 1948 & 19.8 & 1027 & 52.7 \\
\hline Exercise facilities & 3263 & 33.2 & 919 & 28.2 \\
\hline Weekly exercise & 1784 & 18.1 & 457 & 25.6 \\
\hline $\begin{array}{l}\text { Contact to health } \\
\text { professional }\end{array}$ & 3230 & 32.8 & 1260 & 39.0 \\
\hline Health check & 1676 & 17.0 & 752 & 44.9 \\
\hline
\end{tabular}

WHP. WHP offered during leisure time was associated with lower participation in all measured WHP activities compared with when offered during working hours (OR $(\mathrm{CI})=0.70(0.60$ to 0.82$))$ for contact with the health professional, 0.34 (0.26 to 0.43 ) for health screening, 0.75 (0.62 to 0.90 ) for exercise facilities, 0.56 (0.43 to 0.73 ) for weekly exercise, 0.27 ( 0.20 to 0.36$)$ for healthy diet and 0.54 (0.34 to 0.85$)$ for smoking cessation.

\section{Physical work demands}

Having high physical work demands was not associated with WHP participation with ORs ranging from 0.91 to 1.00 for most WHP activities. There were only weak, nonsignificant associations with higher participation in healthy diet ( $\mathrm{OR}=1.23$ (95\% CI 0.97 to 1.54$)$ ) and lower participation in health check $(\mathrm{OR}=0.81(95 \%$ CI 0.66 to $1.01)$ ).

\section{Physical fatigue after work}

Being moderately fatigued after work (compared with no fatigue) was associated with higher participation in contact with the health professional $(\mathrm{OR}=1.25$ (95\% CI 1.03 to 1.51$)$ ) and smoking cessation ( $\mathrm{OR}=1.74$ (95\% CI 1.06 to 2.87)), but not with participation in other WHP activities. Being very fatigued after work was associated with lower participation in weekly exercise sessions ( $\mathrm{OR}=0.54$ (95\% CI 0.31 to 0.94$)$ ) referencing those not being fatigued.

\section{Quantitative demands}

Reporting high quantitative demands at work was not significantly associated with participation in WHP activities, with ORs ranging from 0.83 to 1.13 .

\section{Work pace}

Reporting high work pace was not significantly associated with participation in WHP activities with ORs ranging from 0.88 to 1.02 .

\section{Emotional demands}

Reporting of emotional demands at work was associated with higher participation in contact with health 


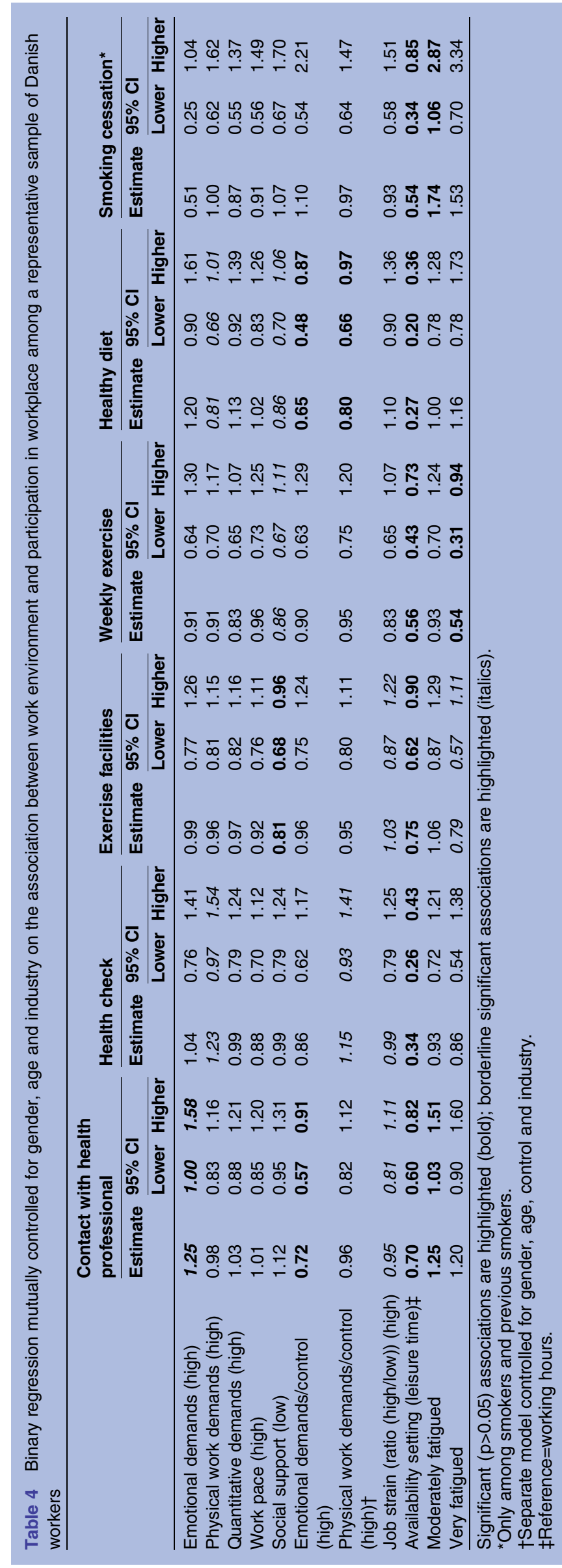

professionals $(\mathrm{OR}=1.25$ (95\% CI 1.0 to 1.58$)$ ). For the other WHP activities, the ORs were non-significant between 0.99 and 1.20. There was a stronger, but also non-significant association between high emotional demands and lower participation in smoking cessation $(\mathrm{OR}=0.51 \quad(95 \%$ CI 0.25 to 1.04$))$, indicating lower participation.

\section{Demands/control ratios}

Demands and control ratios: High job strain was not associated with participation in any WHP activities, with ORs ranging from 0.83 to 1.10 . A high ratio of physical demands to job control was associated with lower participation in healthy diet $(\mathrm{OR}=0.80$ (95\% CI 0.66 to 0.97$)$ ). The physical demands/job control ratio was not associated with other WHP activities, with ORs ranging from 0.95 to 1.15 .

A high ratio of emotional demands to job control was associated with a lower participation in contact with health professionals (OR=0.72 (95\% CI 0.57 to 0.91$)$ ) and healthy diet ( $\mathrm{OR}=0.65$ (95\% CI 0.48 to 0.87$)$ ). This ratio was not significantly associated with any of the other WHP activities with ORs ranging from 0.86 to 1.10 .

\section{Social support}

Low social support was significantly associated with lower participation in exercise facilities $(\mathrm{OR}=0.81 \quad(95 \%$ CI 0.68 to 0.96$)$ ). Low social support was not associated with participation in other WHP activities, with ORs ranging from 0.86 to 1.12 .

\section{DISCUSSION}

In this study of a large representative sample of Danish employees, several characteristics of the work environment were associated with employee participation in WHP. In particular, WHP activities available only during leisure time had markedly lower odds of participation. Adverse work environment factors (ie, low social support and fatiguing work) were also associated with low participation. High demands at work (ie, physical, emotional and quantitative demands) did not seem to be independent a barrier for participation. However, in combination with low control, high physical and emotional work demands seemed to limit participation in WHP. In the following, the results are discussed and compared with previous studies on participation in WHP.

The most dominating factor associated with participation proved to be timing and/or setting of the availability of the WHP activity (ie, during working hours or during leisure time). For all types of health promotion initiatives, being available only in leisure time meant a much lower probability for participation than for availability during working hours. A previous qualitative study suggested that accessibility of WHP was important for WHP participation and suggested that this may be due to the WHP during paid work hours, which signals a 
stronger management commitment. ${ }^{12}$ Moreover, having variable working hours has been shown to impact participation positively. ${ }^{10}{ }^{20}$ Another explanation is that it is simply more feasible for the workers to fit the participation into everyday life (eg, obligations to children or a second job may hamper participation outside working hours). The latter explanation is in line with previous explanations for participation in WHP. For example, the socioecological framework suggests that higher order structural factors are overarching determinants for WHP participation. ${ }^{11}$ In the settings approach suggested by the Ottawa Charter for Health Promotion strategy, location and provider of health promotion were mentioned as important prerequisites for implementation. ${ }^{5}$ However, these additional features were not measured in this study. Furthermore, state-of-the-art implementation techniques also take timing into consideration when mapping barriers and facilitators for implementation of a health education initiative. ${ }^{21}$ Thus, timing seems to be a highly important structural factor to consider when planning and implementing WHP.

The actual production demands at work (emotional, quantitative and physical) were not independently associated with lower participation in WHP. This is in contradiction to previous studies suggesting that a high physical work demands limit smoking cessation and weight loss. ${ }^{14} 15$ However, when combined with a concurrent low level of control, high physical work demands was associated with lower participation in WHP, which is in line with one previous study, indicating lower success in efforts for smoking cessation with high physical job strain. ${ }^{22}$ Specifically, high physical demands combined with low control lowered the odds of participation in healthy diet, and the combination of high emotional demands and low control lowered the odds of contact with health professionals and healthy diet.

The demand-control model is known to be associated with health and well-being and built originally on the concept that high demands and low control could increase the risk of reduced participation in society. ${ }^{4}$ The current study expands that understanding of participation in a way that is logical and plausible but not previously documented. Furthermore, this study established a physical demand-control ratio as previously suggested by Sanderson $e t a l^{22}$ and Schoenfisch and Libscomb. ${ }^{23}$ In addition, an emotional demand-control ratio was established. Similar to the other demand-control ratios, the ratio of emotional demands to control was calculated with emotional demands in the nominator and control in the denominator. The authors are not aware of previous studies that have done this. The association between high emotional demands/control ratio and low participation in contact with health professionals is particularly interesting because emotional demands independently increased the odds of participating in contact with health professionals. One explanation is that high emotional demands at work generate a need to seek help from health professionals ${ }^{16}$ and thus elevates participation propensity, but that low control in the job limits the opportunities for participation and thus lowers participation. However, previously, negative affections have been shown to be highly associated with the selfreporting of psychological job demands, so the results should be interpreted with caution and the finding tested in future studies.

Social support was positively associated with participation in WHP. This is in line with previous studies reporting that social support is a motivator for participation in health promotion. ${ }^{12}{ }^{24}$ Moreover, social support is a wellknown important factor for well-being at work. ${ }^{25}$ Furthermore, the socioecological framework supports the contribution of social support as an interpersonal factor determining participation. ${ }^{11}$ In this study, low social support was associated with low participation in exercise facilities. Exercise facilities do not necessarily include any personal contact with colleagues or other social obligation. Therefore, colleague and supervisor support may become particularly important for participation in such initiatives. Thus, this finding indicates that when offering exercise facilities at the workplace, inclusion of some component of social support should be considered.

One single adverse factor-being moderately fatigued after work-was associated with higher participation in contact with health professionals and smoking cessation. Being fatigued is not per se a feature of the work environment, but rather a short-term indicator of its effect on the person. ${ }^{26}$ The simple fact of acknowledging that one is fatigued may distinguish those who are more willing to seek assistance. Thus, fatigue after work may motivate for individual counselling due to an individually perceived need. Previous analyses of data from the same cohort indicated that some groups of unhealthy employees (ie, individuals with elevated body mass index) do choose to participate in WHP presumably based on their individual motivation to promote their health. ${ }^{16}$ Thus, even though this study indicates that higher order factors in the work environment and organisation are important for participation in WHP, individual motivation may still be an important mediator. ${ }^{13}$ However, reporting of being very fatigued after work was associated with lower participation in weekly exercise. An interpretation of this finding is that high fatigue after work is an important barrier for exercising.

\section{STRENGTHS AND WEAKNESSES}

This study has strengths since it involves a large number of employees from several different occupations and industries in a representative sample of Danish employees. However, the study also includes limitations. First, the selfreported information regarding work environment represents the perceived environment of the participants, which is known to impose risks of bias. For example, negative affect is shown to impact the reporting of psychological work demands. ${ }^{27}$ Likewise, negative affect may be associated with participation in WHP. Such reporting biases 
would render the analyses vulnerable to confounding and reporting bias, which would increase the risk of finding false associations between adverse work environment and low participation. Therefore, the results should be interpreted in the light of this limitation. Second, the selfreported information regarding availability of health promotion represents the perceived availability of health promotion at the workplace. Therefore, it is possible that some workers have health promotion available without being aware of it, and that those aware of health promotion offers may be a selected group of workers. Third, the reporting of participation reflects any level of participation within the last year and thus there may be some misclassification since those participating only once may not be distinguishable from those not participating. In addition, the impact of work environment features on the frequency or intensity of the individual's participation cannot be determined in this study. Finally, all data were collected concurrently and analyses performed in a cross-sectional design. This hampers interpretation of causality. For example, it is possible that participation in WHP can positively impact the perception of the work environment.

\section{Conclusion and implications for clinicians and policymakers}

Adverse work environment factors such as low social support and very fatiguing work were associated with lower employee participation in WHP. High physical, quantitative and emotional demands were not associated with low participation, but high demands in combination with low control lowered the odds for participation in WHP. These findings suggest that to obtain proper implementation and effects of WHP, initiatives for ensuring a good work environment is essential. Furthermore, the probability for participation in WHP seems to be elevated when the activities are offered during paid working hours. Therefore, a supportive work environment seems to be an important foundation for employees' participation in WHP.

\section{Author affiliations \\ ${ }^{1}$ National Research Centre for the Working Environment, Copenhagen, Denmark \\ ${ }^{2}$ Federal Institute for Occupational Safety and Health (BAuA), Berlin, Germany ${ }^{3}$ Department of Work Environment, University of Massachusetts Lowell, Lowell, Massachusetts, USA \\ ${ }^{4}$ Institute of Sports Science and Clinical Biomechanics, University of Southern Denmark, Odense, Denmark}

Contributors MBJ intiated the study and planned the analyses, interpretation and drafting of the manuscript. LP contributed to the planning of the analyses. EV conducted the analyses. $\mathrm{AH}$ and $\mathrm{HB}$ contributed to the interpretation of the results and drafting of the manuscript. All the authors approved of the final version.

Funding This research received no specific grant from any funding agency in the public, commercial or not-for-profit sectors.

Competing interests None declared.

Provenance and peer review Not commissioned; externally peer reviewed.
Data sharing statement The raw data from the survey is publicly available in Danish at the website of the National Research Centre for the Working Environment, Denmark.

Open Access This is an Open Access article distributed in accordance with the Creative Commons Attribution Non Commercial (CC BY-NC 4.0) license, which permits others to distribute, remix, adapt, build upon this work noncommercially, and license their derivative works on different terms, provided the original work is properly cited and the use is non-commercial. See: http:// creativecommons.org/licenses/by-nc/4.0/

\section{REFERENCES}

1. Joint WHO/FAO Expert Consultation on Diet NatPoD. Diet, nutrition and the prevention of chronic diseases. Geneva: World Health Organization, 2003.

2. Holtermann A, Mortensen OS, Burr H, et al. Physical demands at work, physical fitness, and 30-year ischaemic heart disease and all-cause mortality in the Copenhagen Male Study. Scand J Work Environ Health 2010;36:357-65.

3. Kristensen TS. Job stress and cardiovascular disease: a theoretic critical review. J Occup Health Psychol 1996;1:246-60.

4. Theorell T, Karasek RA. Current issues relating to psychosocial job strain and cardiovascular disease research. J Occup Health Psychol 1996;1:9-26.

5. [No authors listed]. The Ottawa Charter for Health Promotion. WHO Reg Publ Eur Ser 1992;44:1-7.

6. Rongen A, Robroek SJW, van Lenthe FJ, et al. Workplace health promotion: a meta-analysis of effectiveness. Am J Prev Med 2013;44:406-15.

7. Robroek SJW, van Lenthe FJ, van EP, et al. Determinants of participation in worksite health promotion programmes: a systematic review. Int J Behav Nutr Phys Act 2009;6:26.

8. Jorgensen MB, Faber A, Jespersen $\mathrm{T}$, et al. Implementation of physical coordination training and cognitive behavioural training interventions at cleaning workplaces - secondary analyses of a randomised controlled trial. Ergonomics 2012;55: 762-72.

9. Hunt MK, Lederman R, Potter S, et al. Results of employee involvement in planning and implementing the Treatwell 5-a-Day work-site study. Health Educ Behav 2000;27:223-31.

10. Kilpatrick M, Blizzard L, Sanderson K, et al. Factors associated with availability of, and employee participation in, comprehensive workplace health promotion in a large and diverse Australian Public Sector setting: a cross-sectional survey. J Occup Environ Med 2015;57:1197-206.

11. Linnan LA, Sorensen G, Colditz G, et al. Using theory to understand the multiple determinants of low participation in worksite health promotion programs. Health Educ Behav 2001;28:591-607.

12. Persson R, Cleal B, Bihal T, et al. Why do people with suboptimal health avoid health promotion at work? Am J Health Behav 2013;37:43-55

13. Hall JL, Kelly KM, Burmeister LF, et al. Workforce characteristics and attitudes regarding participation in Worksite Wellness Programs. Am J Health Promot 2016. [Epub ahead of print 5 Jan 2016].

14. Albertsen K, Hannerz H, Borg V, et al. Work environment and smoking cessation over a five-year period. Scand J Public Health 2004;32:164-71.

15. Hannerz H, Albertsen $\mathrm{K}$, Nielsen ML, et al. Occupational factors and 5 -year weight change among men in a Danish national cohort. Health Psychol 2004;23:283-8.

16. Jorgensen MB, Villadsen $\mathrm{E}$, Burr $\mathrm{H}$, et al. Does workplace health promotion in Denmark reach relevant target groups? Health Promot Int 2015;30:318-27.

17. Grosch JW, Alterman T, Petersen MR, et al. Worksite health promotion programs in the U.S.: factors associated with availability and participation. Am J Health Promot 1998;13:36-45.

18. Garde $\mathrm{AH}$, Albertsen $\mathrm{K}$, Nabe-Nielsen $\mathrm{K}$, et al. Implementation of self-rostering (the PRIO-project): effects on working hours, recovery, and health. Scand J Work Environ Health 2012;38:314-26.

19. de Croon EM, Sluiter JK, Frings-Dresen MHW. Need for recovery after work predicts sickness absence: a 2-year prospective cohort study in truck drivers. J Psychosom Res 2003;55:331-9.

20. Nabe-Nielsen K, Garde AH, Clausen T, et al. Does workplace health promotion reach shift workers? Scand $J$ Work Environ Health 2015;41:84-93.

21. Bartholomew LK, Parcel GS, Kok G. Intervention mapping: a process for developing theory- and evidence-based health education programs. Health Educ Behav 1998;25:545-63. 
22. Sanderson DM, Ekholm O, Hundrup YA, et al. Influence of lifestyle, health, and work environment on smoking cessation among Danish nurses followed over 6 years. Prev Med 2005;41: 757-60.

23. Schoenfisch AL, Lipscomb HJ. Job characteristics and work organization factors associated with patient-handling injury among nursing personnel. Work 2009;33:

$117-28$.

24. Wyatt KM, Brand S, shby-Pepper J, et al. Understanding how healthy workplaces are created: implications for developing a national health service healthy workplace program. Int $J$ Health Serv 2015;45:161-85.
25. Gunnarsdottir S, Bjornsdottir K. Health promotion in the workplace: the perspective of unskilled workers in a hospital setting. Scand $J$ Caring Sci 2003;17:66-73.

26. Sluiter JK, de Croon EM, Meijman TF, et al. Need for recovery from work related fatigue and its role in the development and prediction of subjective health complaints. Occup Environ Med 2003;60(Suppl 1): i62-70.

27. Clausen T, Andersen LL, Holtermann A, et al. Do self-reported psychosocial working conditions predict low back pain after adjustment for both physical work load and depressive symptoms? A prospective study among female eldercare workers. Occup Environ Med 2013;70:538-44. 